\title{
Plectoneme formation in twisted fluctuating rods
}

\author{
Prashant K. Purohit \\ Department of Mechanical Engineering and Applied Mechanics, \\ University of Pennsylvania, Philadelphia, PA 19104, USA.
}

December 3, 2007.

\begin{abstract}
The mechanics of DNA super-coiling is a subject of crucial importance to uncover the mechanism and kinetics of several enzymes. It is therefore being investigated using several biochemical and biophysical methods including single molecule experimental techniques. An interesting problem within this realm is that of torsional buckling and plectoneme formation in DNA as it is simultaneously put under tensile and torsional stress. Analytical solutions to this problem are difficult to find since it involves non-linear kinematics and thermal fluctuations. In this paper we use ideas from the Kirchhoff theory of filaments to find semianalytical solutions for the average shape of the fluctuating DNA under the assumption that there is no self-contact. The basic step in our method consists of combining a helical solution of the rod with a non-planar localizing solution in such a way that the force, moment, position and slope remain continuous everywhere along the rod. Our solutions allow us to predict the extension vs. linking number behavior of long pieces of DNA for various values of the tension and temperature. An interesting outcome of our calculations is the prediction of a sudden change in extension at buckling which does not seem to have been emphasized in earlier theoretical models or experiments. Our predictions are amenable to falsification by recently developed single molecule techniques which can simultaneously track the force-extension as well as the torque-rotation behavior of DNA.
\end{abstract}

Keywords: Buckling, rods, biological material, plectonemes.

Formation of plectonemes in filaments is a commonly observed phenomenon in yarn, hair strands, garden hoses and telephone or computer cords. The process of plectoneme formation is easily demonstrated by holding a string or wire taut by exerting forces at its ends, twisting it and then relieving the tension so that structures of the type shown in figure 1 are formed. In fact, it is now believed that the torque associated with plectoneme formation in the bacterial genome is an important ingredient in the regulation of certain genes (Purohit and Nelson, 2006). Furthermore, several molecular machines manipulate the plectonemes during crucial processes such as transcription and DNA repair (Nelson, 2004). To unravel the mechanics of these machines it is necessary to first understand the response of DNA to torsional moments. This was the goal of some recent single molecule experiments described below.

In a typical experiment a tension $F$ of order $1 \mathrm{pN}$ is applied to a single fluctuating DNA molecule while one of its ends is rotated about an axis coinciding with the direction of the applied force (Strick et al., 1996, 1998, Charvin et al., 2004, Deufel et al., 2007). The end-to-end extension of the molecule is then measured as a function of the number of turns applied to the end. As the number of turns applied is increased the DNA is found to undergo torsional buckling (manifest in a constant post-buckling torque irrespective of the number of turns applied) and plectoneme formation, resulting in a reduction of the end-to-end extension. It is the goal of this work to devise a simple model to interpret experimental data in the context of plectoneme formation in thermally fluctuating DNA. 
In an elegant article Stump and Fraser (see Stump, Fraser and Gates, 1998 and Stump and Fraser, 2000) discussed the mechanics of plectonemes in a rod by dividing it into three regions - the end loop, the ply which consists of two interwound helices in contact, and two tails at whose ends the remote forces and moments are applied (see figure 1). Since there is contact and friction between the helices in the ply there are discontinuities in the force and moment at the boundaries of the three regions and one needs jump conditions to account for these discontinuities. The remaining analysis is carried out by insisting on equilibrium at every point along the rod and continuity of position and tangents at the boundaries of these three regions. The analysis of Stump and Fraser (2000) was extended to variable plys by Coleman and Swigon (see Coleman, Swigon and Tobias, 2000 and Coleman and Swigon, 2000) and further developed by Thompson,van der Heijden and Neukirch (2002) who successfully applied their results to closed DNA rings which were twisted to form plectonemes as in bacterial plasmids. Stump and Fraser (2000) had done a similar comparison between predicted balanced ply shapes and electron micrographs of twisted plasmids and found good agreement. Neukirch (2004) carried out an analysis based on the method of Stump and Fraser (2000) for determining the forceextension response of DNA under torsional moments and was able to fit the post-buckling response data (Strick et al., 1996) for number of turns vs. extension quite well. All of these analyses accounted for self-contact of the rod (see also van der Heidjen et al., 2003 and Starostin, 2004 for analytical solutions of rod equilibria with self-contact) but none of them considered the effects of fluctuations (which are important for DNA, especially in the context of force-extension measurements) but were still able to obtain good agreement with experiment since the scales of bending and twisting energies involved in plectoneme formation are much larger than the scale of thermal energy. The methods presented in this paper differ from this body of work in that we account for the effect of fluctuations (or configurational entropy) in an effort to make contact with force-extension measurements.

The effects of Brownian motion in twisted rods was first treated by Moroz and Nelson (see Moroz and Nelson, 1997, 1998) under the assumption that the shape fluctuations caused by thermal motion are small enough that the energy can be approximated by expanding upto quadratic order around a straight equilibrium configuration. This assumption holds good until the rod buckles under the action of a force and moment. Moroz and Nelson (1997) applied their model to extract a twisting modulus for DNA under tension from the experimental data of Strick et al. (1996) which measured the end-to-end extension of a long piece of DNA as a function of the added link at various values of the applied tension as described above. Their analysis was restricted to the pre-buckling regime and built on prior work of Marko and Siggia (1995) and Odijk (1995) who developed force-extension relations for thermally fluctuating rods with bending alone and no twist. An early attempt to account for twist in a fluctuating rod was that of Fain, Rudnick and Ostlund (1997) who recognised that the entropic part of the free energy of the plectonemes is negligible in comparison to the elastic energy so that most of the entropy comes from the straight portions of the rod. We follow their line of thought in this paper but our results differ from theirs in that we can explicitly predict the sudden change in extension of the rod as it buckles, and moreover, our treatment (which follows the work of Moroz and Nelson, 1997, 1998) of the straight portion of the rod includes softening of the twist rigidity due to thermal motion, which they do not account for. Our results also differ from those of Moroz and Nelson $(1997,1998)$ since we are able to capture both the pre- and post-buckling response of the DNA.

As in all of the work described above we treat the DNA as a continuous, inextensible, 
isotropic elastic rod, with a linear relation between the moment and curvature. We neglect bending anisotropy, non-linear DNA elasticity and strand separation in the present treatment while acknowledging that they are likely to be important in the quantitative understanding of plectoneme formation in DNA. We also neglect electrostatic effects or more precisely, assume that they can be incorporated via effective values of the bending and twisting moduli. The advantage of these simplifications is that they will allow us to use the elegant closed-form solutions (see Nizette and Goriely, 1999 and references therein) to the elastic equilibrium equations. These solutions will be combined with some known analytical results on the entropic elasticity of fluctuating DNA to model the phenomena observed in some recent experiments on long DNA molecules where thermal motion results in significant configurational entropy.

\section{Review of Kirchhoff's theory of rods}

In the following we briefly review Kirchhoff's theory for the equilibrium of rods and some known analytical solutions which we will use in our development. The coordinates of the center-line of a Kirchhoff rod are given by a position vector $\mathbf{P}(s)$ where $s$ is the arc-length along the rod. At each point $s$ of the rod we attach a coordinate frame called the material frame. The unit vectors of the material frame are denoted by $\mathbf{d}_{1}(s), \mathbf{d}_{2}(s), \mathbf{d}_{3}(s)$ with $\mathbf{d}_{3}(s)$ chosen to be along the tangent to the rod at every point so that $\mathbf{d}_{3}(s)=\mathbf{P}^{\prime}(s)=\frac{d \mathbf{P}(s)}{d s}$. Note that the tangent $\mathbf{P}^{\prime}(s)$ is a unit vector since the rod is assumed to be inextensible. The derivatives of these vectors along the arc-length $s$ contain information about the local curvature of the rod. More explicitly, we note that

$$
\frac{d \mathbf{d}_{i}}{d s}=\kappa \times \mathbf{d}_{i}, \quad \text { for } \quad i=1,2,3,
$$

where $\kappa(s)=\left[\begin{array}{lll}\kappa_{1}(s) & \kappa_{2}(s) & \kappa_{3}(s)\end{array}\right]$ is the curvature vector. The moment $\mathbf{M}(s)$ at any point on the rod is given by

$$
\mathbf{M}=K_{b} \kappa_{1} \mathbf{d}_{1}+K_{b} \kappa_{2} \mathbf{d}_{2}+K_{t} \kappa_{3} \mathbf{d}_{3},
$$

where $K_{b}$ and $K_{t}$ are the bending and twisting moduli of the DNA. The equilibrium equations for the rod are then simply,

$$
\frac{d \mathbf{F}}{d s}=\mathbf{0}, \quad \frac{d \mathbf{M}}{d s}+\mathbf{d}_{3} \times \mathbf{F}=\mathbf{0},
$$

where $\mathbf{F}(s)$ is the internal force in the rod. These equations imply that $\mathbf{F}(s)$ is constant along the rod and its value is determined by the external force applied on the DNA, which in the experiments considered in this paper are applied using an optical tweezer setup (Deufel et al., 2007). Following Nizette and Goriely (1999) we will assume that the laboratory coordinate frame is chosen in such a way as the applied force $\mathbf{F}$ is aligned with the z-axis so that $\mathbf{F}(s)=F \mathbf{e}_{z}$. The position vector $\mathbf{P}(s)$ is then explicitly written as

$$
\begin{aligned}
\mathbf{P}(s) & =X(s) \hat{\mathbf{e}}_{x}+Y(s) \hat{\mathbf{e}}_{y}+Z(s) \hat{\mathbf{e}}_{z} \\
& =R(s) \cos \Phi(s) \hat{\mathbf{e}}_{x}+R(s) \sin \Phi(s) \hat{\mathbf{e}}_{y}+Z(s) \hat{\mathbf{e}}_{z},
\end{aligned}
$$

where we have used a cylindrical coordinate system. We will look for solutions with the following symmetry since the buckled and plectonemic shapes of the rod have this symmetry (as we will see later).

$$
X(s)=-X(-s), \quad Y(s)=Y(-s), \quad Z(s)=-Z(-s) .
$$


This suggests that $\Phi(s)$ is of the form $\Phi(s)=\frac{\pi}{2}-\alpha(s)$ with $\alpha(-s)=-\alpha(s)$.

The known analytical solutions of the equlibrium equations for $R(s), \Phi(s)$ and $Z(s)$ are summarised in Nizette and Goriely (1999). There are four constants in the solution $-z_{1}, z_{2}$, $z_{3}$ and $\lambda$ (see also Purohit and Nelson, 2006). The constant $\lambda$ is related to the force through $\frac{K_{b}}{F}=\lambda^{2}$. The other constants $z_{1}, z_{2}$ and $z_{3}$ are related to the three components of the moment M. These three constants can be determined by enforcing the boundary conditions, which leads to a set of algebraic equations that must be solved numerically (e.g., by Newton's method).

\section{Post-buckled shapes of a rod}

The problem of stability of a straight rod has been thoroughly investigated in the literature (see for example, Maddocks (1984) and references therein). A crucial result obtained through a linear stability analysis around a straight configuration shows that the straight twisted configuration becomes unstable (Love, 1944) to small perturbations at the critical twist density $\frac{2 \sqrt{K_{b} F}}{K_{t}}$ where $F$ is the applied tension. When the applied twist density reaches this critical value the rod buckles and the torque immediately falls to $M_{3}<2 \sqrt{K_{b} F}$ so that the link which was entirely in the form of twist in the straight rod is now stored partly as twist and partly as writhe of the nonplanar buckled shape. The linear analysis does not allow us to compute the post-buckled shape of the rod, but the shapes can be readily determined using the known analytical expressions for the fully non-linear rod equilibria. In this section we illustrate this for small forces (small enough so that the critical link at buckling $\frac{2 L \sqrt{K_{b}} F}{K_{t}}<2 \pi$ ) by setting $z_{2}=1$ while $z_{1}, z_{3}$ and $\lambda$ are chosen such that $-1 \leq z_{1} \leq 1$ and $z_{3}>1$. The more general case of $\frac{2 L \sqrt{K_{b} F}}{K_{t}}>2 \pi$ which leads to plectoneme formation is discussed in the next section.

The general solution summarised in Nizette and Goriely (1999) can be specialised to the case of $z_{2}=1$ to yield:

$$
\begin{aligned}
Z(s) & =z_{3} s-\lambda\left(z_{3}-z_{1}\right) E\left(\frac{s}{\lambda} \mid k\right), \\
Z^{\prime}(s) & =z_{1}+\left(1-z_{1}\right) \operatorname{sn}^{2}\left(\frac{s}{\lambda} \mid k\right), \\
\frac{M_{3} \lambda}{K_{b}}=\frac{M_{z} \lambda}{K_{b}} & =\sqrt{\left(1+z_{1}\right)\left(1+z_{3}\right)},
\end{aligned}
$$

where $k^{2}=\frac{1-z_{1}}{z_{3}-z_{1}}, \operatorname{sn}(u \mid k)$ is an elliptic function (see below) and $E(u \mid k)$ is the incomplete elliptic integral of the second kind defined as follows (for details, see Abramowitz and Stegun, 1964):

$$
E(u \mid k)=\int_{0}^{u}\left(1-k^{2} \operatorname{sn}^{2}(x \mid k)\right) d x
$$

where

$$
\operatorname{sn}(x \mid k)=\sin \varphi, \quad \text { and } \quad x=\int_{0}^{\varphi} \frac{d \theta}{\sqrt{1-k^{2} \sin ^{2} \theta}} .
$$

In the solution given above $\lambda=\sqrt{\frac{K_{b}}{F}}$ is determined immediately since the force $F$ is prescribed. The remaining constants $z_{1}$ and $z_{3}$ are determined from the known value of the applied torque $M_{3}$ and the condition that $Z^{\prime}\left( \pm \frac{L}{2}\right)=1$ which says that the tangents at the two ends of the rod are aligned with the z-axis in the laboratory frame which we recall is chosen in such a way as 
$\mathbf{F}=F \mathbf{e}_{z}$. The resulting boundary condition is

$$
1=z_{1}+\left(1-z_{1}\right) \operatorname{sn}^{2}\left(\frac{L}{2 \lambda} \mid k\right) .
$$

This equation has two solutions $-z_{1}=1$, which corresponds to the pre-buckled straight rod, and $\operatorname{sn}^{2}\left(\frac{L}{2 \lambda} \mid k\right)=1$, which corresponds to the post-buckled bent and twisted shape. For the straight $\operatorname{rod} z_{1}=1$ implies $k^{2}=0$ and hence $Z\left( \pm \frac{L}{2}\right)= \pm \frac{L}{2}$ irrespective of the value of $z_{3}$ which is determined from the applied torque $M_{3}$ through $M_{3}=\frac{\sqrt{2} K_{b}}{\lambda} \sqrt{1+z_{3}}$. For the solution corresponding to the post-buckled state we recall that $\operatorname{sn}^{2}\left(\frac{L}{2 \lambda} \mid k\right)=1$ implies that $\frac{L}{2 \lambda}=(2 n-1) K(k)$ where $K(k)$ is the complete elliptic integral of the first kind with modulus $k$ and $n>0$ is an integer. The lowest energy solution corresponds to $n=1$. The configuration of the rod is determined by solving the following equations (using Newton's method) for $\lambda, z_{1}$ and $z_{3}$.

$$
\lambda=\sqrt{\frac{K_{b}}{F}}, \quad M_{3}=\frac{K_{b}}{\lambda} \sqrt{\left(1+z_{1}\right)\left(1+z_{3}\right)}, \quad k^{2}=\frac{1-z_{1}}{z_{3}-z_{1}}, \quad \frac{L}{2 \lambda}=(2 n-1) K(k) .
$$

The last of these equations sets the value of $k$ once $\lambda$ is determined from the force using $\lambda=\sqrt{\frac{K_{b}}{F}}$. The value of $k^{2}$ therefore remains constant as $M_{3}$ varies, and the end-to-end distance is determined using

$$
Z\left(\frac{L}{2}\right)-Z\left(-\frac{L}{2}\right)=z_{3} L-2 \lambda\left(z_{3}-z_{1}\right) E(k)=\frac{L}{k^{2}}+z_{1} L\left(1-\frac{1}{k^{2}}\right)+2 \lambda E(k) \frac{z_{1}-1}{k^{2}},
$$

where $K(k)$ and $E(k)$ are the complete elliptic integrals of the first and second kind respectively. We plot the non-dimensionalized extension $\zeta=\frac{Z\left(\frac{L}{2}\right)-Z\left(-\frac{L}{2}\right)}{L}$ as a function of the nondimensionalized applied torque for various values of the tension $F$ in figure 2. From the figure it is immediately apparent that the extension $\zeta$ is minimum when $M_{3}=0$ (corresponding to $z_{1}=-1$ ) and $\zeta \rightarrow 1$ (its maximum value) as the applied torque $M_{3} \rightarrow 2 \sqrt{K_{b} F}$. As the torque $M_{3}$ is increased from 0 to $2 \sqrt{K_{b} F}, z_{3}$ decreases monotonically from $\frac{2}{k^{2}}+1$ to 1 (see figure 3 ), and $z_{1}$ increases monotonically from -1 to 1 implying that (see (13) above) the rod is progressively straightened. The case $z_{1}=-1, z_{2}=1, z_{3}=1$ corresponds to the well-known planar homoclinic loop solution (with $k^{2}=1$, see Nizette and Goriely, 1999) for which we see that the change in extension $1-\zeta$ scales as $\lambda$ or $F^{-1 / 2}$. In figure 3 we plot how $z_{3}$ varies as a function of $M_{3}$ for three different values of the length $L$ and bending and twisting moduli typical of DNA. It is clear that $z_{3} \approx 1$ for all values of $M_{3}$ for this set of parameters and furthermore $\left(z_{3}-1\right) \rightarrow 0$ as the length $\frac{L}{2 \lambda}$ becomes larger. Said differently, as the length $L$ becomes longer our solutions tend to the well known non-planar localizing solutions of Coyne (see Nizette and Goriely, 1999) in which $z_{2}=z_{3}=1$ and and $z_{1}$ is such that $-1 \leq z_{1} \leq 1$. The parameter $k^{2}=1$ for the Coyne solution and hence the boundary condition (11) will be violated for a rod of finite length ( since $\frac{L}{2 \lambda}=(2 n-1) K(k) \rightarrow \infty$ as $k^{2} \rightarrow 1$ ). This means that the Coyne solution does not describe the shape of a buckled rod of finite length in a rigorous sense, but in practise it is a good approximation in the limit where $\frac{L}{2 \lambda}$ is large as in the optical or magnetic tweezer experiments on DNA (Strick et al., 1996, 1998 and Deufel et al., 2007) where typically $L \approx 1 \mu \mathrm{m}$ and $F \approx 1 \mathrm{pN}$. We will combine the Coyne solution with helical solutions in the next section to describe plectoneme formation in twisted DNA. 


\section{Plectoneme formation}

As we add more link at the end of the rod after buckling it forms plectonemes. We think of the plectonemic region as two helices with their axes pointing along the y-direction. The helices are reflections of each other across the y-axis. Consider any one of those helices. On one end of the helix is the part of the rod where the remote force (and torque or link) is applied and on the other end is a loop. For the purposes of our analysis in this section we make the simplifying assumption that the helices have an integer number of turns ${ }^{1}$ so that if the helices were removed and the remaining portion of the rod and end loop were glued together we would obtain a shape of the type discussed in the previous section. Note that there will be no discontinuity in tangents and curvatures at the point where the loop and tail portions are joined together since we assumed an integer number of turns in the helices which we know are constant curvature solutions of the equilibrium equations. The ideas outlined above will now be used for obtaining plectonemic shapes of torsionally buckled rods. We will combine the helical solutions with other known solutions of the rod by enforcing continuity of the material frame unit vectors, forces and moments at the boundaries of the helices. We emphasize that this method based on combining helices with other known solutions by imposing continuity is not new and has been used by other authors both in the context of the mechanics of yarn and also in the mechanics of twisted DNA plasmids as reviewed in an earlier section. The chief distinguishing feature of the solutions presented here is that unlike the aforementioned work we do not have contact or friction between the helices leading to equations that can be solved without recourse to sophisticated numerics.

To begin let us assume that the total length of the rod is $L$ and the length eaten up by the helical region is $2 L_{2}$ so that $L_{1}=L-2 L_{2}$ is the leftover length that (without the helices) corresponds to a shape described in the previous section. At present $L_{1}$ is an unknown and so are $\pm s_{1}$ (with $-\frac{L_{1}}{2}<-s_{1}<0<s_{1}<\frac{L_{1}}{2}$ ) which are the arclengths at which we cut the rod to insert the plectonemic helices. The arclength interval $\left[-s_{1}, s_{1}\right]$ corresponds to the end loop referred to above while the intervals $\left[-\frac{L_{1}}{2},-s_{1}\right]$ and $\left[s_{1}, \frac{L_{1}}{2}\right]$ correspond to the tails with the ends $s= \pm \frac{L_{1}}{2}$ where the tension $F$ is applied. The center-line of the helix is given by $\mathbf{P}(s)=\left[\begin{array}{lll}r \cos (A s+B) & Q s+D & r \sin (A s+B)\end{array}\right]$ where $A, B, Q, D$ and $r$ are all constants. The tangent vector to the helix is simply $\mathbf{P}^{\prime}(s)=\left[\begin{array}{lll}-A r \sin (A s+B) & Q & A r \cos (A s+B)\end{array}\right]$ where we require $A^{2} r^{2}+Q^{2}=1$. The length of each of the helices is $L_{2}=2 \pi m r \sec \alpha$ where $\alpha$ is the pitch angle $\left(\tan \alpha=\frac{Q}{A r}\right)$ and $m$ is the number of turns in each helix. Fuller (1971) showed that the writhing number for this plectonemic solution (completed to a closed curve) is $2 m \sin \alpha$. Assuming that $\kappa_{3}=\frac{M_{3}}{K_{t}}$ is the constant twist rate in the rod in the post-buckled shape, the total link stored in the rod can be obtained from the Fuller-White-Calugrenau relation which is equated to the prescribed change in link $\Delta L k$ leading to:

$$
\begin{aligned}
\Delta L k & =\frac{M_{3} L}{2 \pi K_{t}}+2 m \sin \alpha, \\
L & =L_{1}+4 \pi m r \sec \alpha
\end{aligned}
$$

where the second equation merely says that the length in the helices and the rod solution should add up to the total length $L$.

\footnotetext{
${ }^{1}$ Our method will also work if each helix had $n+\frac{1}{2}$ turns where $n$ is an integer but then we would have to rotate the loop in the interval $\left[-s_{1}, s_{1}\right]$ by $\pi$ radians about the $\mathrm{y}$-axis. The rigid body rotation preserves the assumed symmetry of the solution and it also does not violate equilibrium.
} 
The curvature vector of the helix is $\mathbf{P}^{\prime \prime}(s)=\left[\begin{array}{llll}-A^{2} r \cos (A s+B) & 0 & -A^{2} r \sin (A s+B)\end{array}\right]$. From these we infer that the Frenet curvature and torsion (Nizette and Goriely, 1999) of the helix are respectively $\kappa=A^{2} r$ and $\tau=A Q=A \sqrt{1-A^{2} r^{2}} . s_{1}$ is the arclength at which the rod solution intersects the helix. We require continuity of the tangent, the force and the moment at this point. We will denote the helical side by $s_{1}^{+}$and the rod side by $s_{1}^{-}$. Continuity of the position vector and tangent vector at this point imply:

$$
\begin{aligned}
X\left(s_{1}^{-}\right)=r \cos \left(A s_{1}^{+}+B\right), & Y\left(s_{1}^{-}\right)=Q s_{1}^{+}+D, \quad Z\left(s_{1}^{-}\right)=r \sin \left(A s_{1}^{+}+B\right),(16) \\
X^{\prime}\left(s_{1}^{-}\right)=-A r \sin \left(A s_{1}^{+}+B\right), \quad Y^{\prime}\left(s_{1}^{-}\right) & =Q, \quad Z^{\prime}\left(s_{1}^{-}\right)=A r \cos \left(A s_{1}^{+}+B\right)
\end{aligned}
$$

These can be satisfied if we find a point $s_{1}$ in the rod solution with $0<s_{1}<L_{1}$, such that

$$
X\left(s_{1}\right) X^{\prime}\left(s_{1}\right)+Z\left(s_{1}\right) Z^{\prime}\left(s_{1}\right)=\left.\frac{d}{d s}\left(X^{2}(s)+Z^{2}(s)\right)\right|_{s_{1}}=0 .
$$

If there exists such a point in the rod solution then the parameters $A, B, D, Q$ and $r$ for the helix can be uniquely determined. We see that $s_{1}$ is simply the arc-length at which the distance $\sqrt{X^{2}(s)+Z^{2}(s)}$ is a minimum. Intuitively, we know there is such a location $s_{1}$ in the rod solution and therefore there exists an $s_{1}$ which will satisfy (18) above. Once we find $s_{1}$ the parameters of the helix are determined using

$$
r^{2}=X^{2}\left(s_{1}\right)+Z^{2}\left(s_{1}\right), \quad Q=Y^{\prime}\left(s_{1}\right), \quad A^{2}=\frac{1-Y^{\prime 2}\left(s_{1}\right)}{X^{2}\left(s_{1}\right)+Z^{2}\left(s_{1}\right)} .
$$

We also need to satisfy continuity of forces and moments at $s_{1}$. For a helix the force and moment equilibrium equations can be summarised by a single vector equation written below in the material frame (see Nizette and Goriely, 1999).

$$
\mathbf{F}=\left(K_{t} \kappa_{3}-K_{b} \tau\right)\left[\kappa_{1} \mathbf{d}_{1}+\kappa_{2} \mathbf{d}_{2}+\tau \mathbf{d}_{3}\right]
$$

where $\mathbf{d}_{3}(s)=\mathbf{P}^{\prime}(s)$ is the tangent vector. Taking magnitudes on both sides of the equation above we get

$$
F=\left(K_{t} \kappa_{3}-K_{b} \tau\right) \sqrt{\kappa_{1}^{2}+\kappa_{2}^{2}+\tau^{2}} .
$$

Now, for any equilibrium solution of a Kirchhoff rod we know that $M_{3}$ and $M_{z}$ are constants independent of $s$. We exploit this fact in the helical solution and note that

$$
\left|K_{b} \kappa_{1} \mathbf{d}_{1}+K_{b} \kappa_{2} \mathbf{d}_{2}\right|^{2}=\left|\mathbf{M}-M_{3} \mathbf{d}_{3}\left(s_{1}\right)\right|^{2}=M_{3}^{2}\left|\mathbf{e}_{z}-\mathbf{d}_{3}\left(s_{1}\right)\right|^{2}=2 M_{3}^{2}\left(1-Z^{\prime}\left(s_{1}\right)\right) .
$$

Substituting this result back into (21) and remembering that the force and moment have to be continuous at $s_{1}$ we obtain

$$
F=\left(M_{3}-K_{b} \tau\right) \sqrt{\frac{2 M_{3}^{2}}{K_{b}^{2}}\left(1-Z^{\prime}\left(s_{1}\right)\right)+\tau^{2}},
$$

which after some re-arrangement can be recast as

$$
1=\left(\frac{M_{3} \lambda}{K_{b}}-\tau \lambda\right) \sqrt{\frac{2 M_{3}^{2} \lambda^{2}}{K_{b}^{2}}\left(1-Z^{\prime}\left(s_{1}\right)\right)+\tau^{2} \lambda^{2}} .
$$


Equations (14), (15), (18) and (24) together with the following are six equations for the six unknowns $-L_{1}, s_{1}, m, z_{1}, z_{3}$ and $M_{3}$.

$$
\frac{M_{3} \lambda}{K_{b}}=\sqrt{\left(1+z_{1}\right)\left(1+z_{3}\right)}, \quad \frac{L_{1}}{2 \lambda}=K\left(\frac{1-z_{1}}{z_{3}-z_{1}}\right) .
$$

For instance, if $\frac{L}{2 \lambda}$ is large we could assume $z_{3}=1$ (see previous section) and solve (18) and (24) for $\frac{s_{1}}{\lambda}$ and $z_{1}$. The scaled helix parameters $A \lambda, Q$ and $\frac{r}{\lambda}$ can then be determined immediately from (19). For a given value of $F$ and material properties $K_{b}$ and $K_{t}$, the helix parameters $A$, $Q, r$ etc., can then be obtained and $L_{1}$ and $m$ can be determined using (14) and (15). This completely determines a plectonemic shape.

\subsection{Plectonemes in DNA under tension}

We consider now an example inspired by recent single molecule experiments on plectoneme formation in DNA. We assume that $L=750 \mathrm{~nm}$ and the mechanical properties of DNA are summarised by $K_{b}=205 \mathrm{pNnm}^{2}$ and $K_{t}=431 \mathrm{pNnm}^{2}$ (see for example, Moroz and Nelson, 1997). The critical torque for buckling (in the absence of thermal fluctuations) is $2 \sqrt{K_{b} F}=$ $40.5 \mathrm{pNnm}$ for $F=2 \mathrm{pN}$, which for a rod of length $L=750 \mathrm{~nm}$ corresponds to a critical link $\Delta L k_{c}=\frac{2 L \sqrt{K_{b} F}}{2 \pi K_{t}}=11.23$ turns. In other words, the DNA should remain straight until 11.23 turns are added and then buckle. Experimental evidence (personal communication with Wang) indicates that this is not the case and the DNA buckles at a lower value of about 8 turns for $F=2 \mathrm{pN}$ which corresponds to a critical torque of $\sqrt{2 K_{b} F}$. This lower value of critical buckling torque can also be explained theoretically if one accounts for thermal fluctuations of the rod and then calculates the critical torque by finding the configuration which has the minimum free-energy (Moroz and Nelson, 1998). We would like to determine the end-to-end distance before and after buckling since this is one of the observables in recent experiments. To this end we assume $z_{3}=1$ and solve (18) and (24) to obtain $z_{1}, \frac{s_{1}}{\lambda}$ etc., and ultimately the lengths $L_{1}, L_{2}, r$ etc. The results are plotted in figure 4 as a funtion of the force $F$. A striking feature of the results is that the length $2 L_{2}$ which is the length eaten up by the plectonemic region immediately after buckling stays nearly constant at $196.4 \mathrm{~nm}$. Note that this implies that $L_{1}=750-196.4=553.6 \mathrm{~nm}$ also stays nearly constant irrespective of the force $F$. For this value of $L_{1}$ we are justified in assuming $z_{3}=1$ as explained in the previous section and summarised in figure 3. The radius of the helices decreases with increasing force as might be expected intuitively and the values of the radius are in excess of $4 \mathrm{~nm}$. We recall from (18) that the radius of the helix is determined from the minimum value of $X^{2}(s)+Z^{2}(s)$ along the rod and hence conclude that the assumption of no self-contact of the rod is also not violated since we know that the radius of DNA is about $1 \mathrm{~nm}$ (Nelson, 2004).

When we add more turns after the rod has buckled to form plectonemes $m$ increases linearly with the number of turns added since $\kappa_{3}$ does not change. In other words the length

of the plectonemes increases at the expense of the end-to-end distance $Z\left(\frac{L_{1}}{2}\right)-Z\left(-\frac{L_{1}}{2}\right)$. The length of the plectoneme is linear in $m$ which in turn varies linearly with the number of turns added. Hence, the end-to-end distance after buckling decreases approximately linearly with the number of turns added. This is clearly seen in figure 5 where we have plotted the end-to-end distance $v s$. number of turns for three different values of the force $F$. The qualitative features observed in recent experiments (Strick et al., 1996, 1998, Deufel et al., 2007, Wang (personal communication)) on DNA are neatly captured by our model. The slope of the linear portion of 
the curves after buckling depends on the value of the tension $F$ since this slope is determined by $\kappa_{3}$ which depends on $F$. A dramatic feature of the curves shown in figure 5 is that the change in end-to-end distance at buckling remains the same no matter what $F$ is. This is to be expected since $L_{1}$ remains independent of the force as shown in figure 4 . A caveat of figure 5 is that it does not represent the correct post-buckling response for end-to-end distances less than about $150 \mathrm{~nm}$ since the assumption $z_{3}=1$ used to obtain the curves is violated in this regime. But, the framework developed in this section does not rely on this assumption and similar computations can be performed without enforcing $z_{3}=1$ as was done in the previous section and outlined above. Also, the presence of thermal fluctuations in experiments on DNA changes the picture completely and renders figure 5 irrelevant in their context in spite of capturing many of the qualitative features. In the next section we will explore a simple method to incorporate the effect of thermal fluctuations and also dispense with the assumption of $z_{3}=1$ which was used above primarily to simplify computations.

\section{Thermal fluctuations}

In the absence of thermal fluctuations a straight rod remains straight when the curvature $\kappa_{3}$ is such that $\kappa_{3} L<2 \pi \Delta L k_{c}$ where $\Delta L k_{c}$ is the critical link to cause buckling. Before buckling all the link is stored in the rod as twist since a straight rod does not have any writhe. However, if the shape of the rod is fluctuating around a straight equilibrium configuration the link is stored in the rod partly as twist and partly as writhe in accordance with the Fuller-White-Calugrenau relation. This is the first point of departure from the well understood zero temperature torsion of a rod. Moreover, the inextensibility of the rod results in the end-to-end distance not being equal to $L$ as was the case in the absence of thermal fluctuations. In fact, the end-to-end distance for a fluctuating rod depends on the applied tension $F$ as well as link added as was demonstrated in the beautiful experiments of Strick et al. (1996). This is the second point of departure from the zero temperature case and we see that the problem of torsion of a fluctuating rod is far more complex than the zero temperature case even if we do not consider torsional buckling.

Fortunately, Moroz and Nelson (1997) found a way to determine the pre-buckling torsional response of a fluctuating rod in the limit when fluctuations are small. In this limit which is appropriate for tensions $F>0.5 \mathrm{pN}$ the elastic energy of the rod can be expanded upto quadratic order in the fluctuations so that the resulting thermal averages (within a constant temperature ensemble) can be represented as Gaussian path integrals which can be computed exactly. Their results (see Moroz and Nelson, 1997, 1998) made it possible to accurately determine the twisting modulus $K_{t}$ of DNA. In fact the number $K_{t}=431 \mathrm{pNnm}^{2}$ used throughout this paper was for the first time reported by them after fitting the model to the data of Strick et al., (1996). The expressions derived by Moroz and Nelson (1997) are specialised to an inextensible rod (by assuming the linear stretch constant $\gamma$ in their expressions to be infinite) and summarised as follows:

$$
\begin{aligned}
M_{3}(F, \Delta L k) & =\frac{2 \pi \Delta L k}{\frac{L}{K_{t}}+\frac{L k_{B} T}{4 K_{b} \sqrt{K_{b} F}}}, \\
\zeta(F, \Delta L k) & =1-\frac{1}{2} \frac{1}{\sqrt{\frac{K_{b} F}{k_{B}^{2} T^{2}}-\frac{M_{3}^{2}}{4 k_{B}^{2} T^{2}}-\frac{1}{32}}}+\frac{K_{b} k_{B} T}{L\left(K_{b} F-\frac{M_{3}^{2}}{4}\right)},
\end{aligned}
$$


where $\zeta=\frac{Z\left(\frac{L}{2}\right)-Z\left(-\frac{L}{2}\right)}{L}$ is the non-dimensionalized extension, $k_{B}$ is Boltzmann's constant and $T$ is the absolute temperature. The above expressions are valid as long as $M_{3}<2 \sqrt{K_{b} F}$ which, we recall, is the classical critical buckling torque in the zero temperature limit. A few comments about the above results are in order. First, we see from (26) that fluctuations result in a softening of the rod which is proportional to the temperature $T$ since the fluctuations around equilibrium are larger for higher temperatures. In fact, the classical expression for the torque $M_{3}=K_{t} \frac{2 \pi \Delta L k}{L}$ for a straight rod is recovered from (26) when we set $T=0$ which corresponds to no fluctuations. Fluctuations can also be supressed by applying larger tensions $F$ resulting in lesser softening and this too is borne out in (26). Equation (26) says that the softening occurs because the link is now stored partly as twist and partly as writhe (as explained above) so that we now have two 'torsional springs' in series whose stiffness is the harmonic mean of the original stiffnesses of the springs $-\frac{K_{t}}{L}$ and $\frac{4 K_{b} \sqrt{K_{b} F}}{L k_{B} T}$. The stiffness $\frac{4 K_{b} \sqrt{K_{b} F}}{L k_{B} T}$ of the 'spring' storing link as writhe involves the bending modulus $K_{b}$ since writhe is a manifestation of the out of plane bending of the rod. Equation (27) shows how an experimental observable, namely the end-to-end distance, varies as a function of the applied tension and link. In particular, when $M_{3}=0$ we find that $\zeta \approx 1-\frac{1}{2} \frac{k_{B} T}{\sqrt{K_{b} F}}+\frac{k_{B} T}{F L}$ which is the well-known result of Marko and Siggia (1995) and Odijk (1995) for the so called worm-like-chain (WLC) model of a fluctuating rod which assumes that rod deforms only in bending and not in torsion. It is also apparent from this expression that $\zeta=\frac{Z\left(\frac{L}{2}\right)-Z\left(-\frac{L}{2}\right)}{L}=1$ when $T=0$ as is expected for the equilibrium of a purely elastic rod.

We will use (26) and (27) in the straight portions of the buckled rod, meaning for those $s$ where the tangent $\mathbf{P}^{\prime}(s) \approx \mathbf{e}_{z}$ in the zero-temperature equilibrium solution. This leaves the question of how to account for the thermal fluctuations around the plectonemic helices which form after buckling of the rod. Here we will follow the approach of Fain, Rudnick and Ostlund (1997) who showed that the flcutuations in plectonemes make a negligible contribution to the free energy of a buckled rod. As a result the buckled fluctuating rod can be viewed as consisting of three regions - two largely straight but highly fluctuating regions corresponding to arc-length intervals $\left[-\frac{L}{2},-\frac{L_{1}}{2}\right]$ and $\left[\frac{L_{1}}{2}, \frac{L}{2}\right]$ and a highly curved region corresponding to $\left[-\frac{L_{1}}{2}, \frac{L_{1}}{2}\right]$ plus the plectonemic helices with negligible fluctuations. We will use (26) and (27) in the intervals $\left[-\frac{L}{2},-\frac{L_{1}}{2}\right]$ and $\left[\frac{L_{1}}{2}, \frac{L}{2}\right]$ and apply ideas from section 3 to the interval $\left[-\frac{L_{1}}{2}, \frac{L_{1}}{2}\right]$. Following the development of section 3 the following equations need to be satisfied:

$$
\begin{aligned}
X\left(s_{1}\right) X^{\prime}\left(s_{1}\right)+Z\left(s_{1}\right) Z^{\prime}\left(s_{1}\right) & =0, \\
\left(\frac{M_{3} \lambda}{K_{b}}-\tau \lambda\right) \sqrt{\frac{2 M_{3}^{2} \lambda^{2}}{K_{b}^{2}}\left(1-Z^{\prime}\left(s_{1}\right)\right)+\tau^{2} \lambda^{2}} & =1, \\
\sqrt{\left(1+z_{1}\right)\left(1+z_{3}\right)} & =\frac{M_{3} \lambda}{K_{b}}, \\
K\left(\frac{1-z_{1}}{z_{3}-z_{1}}\right) & =\frac{L_{1}}{2 \lambda}, \\
\frac{M_{3} L}{2 \pi K_{t}}+\frac{M_{3} L_{f} k_{B} T}{8 \pi K_{b} \sqrt{K_{b} F}}+2 m \sin \alpha & =\Delta L k, \\
L_{f}+L_{1}+4 \pi m r \sec \alpha & =L,
\end{aligned}
$$

where the parameters $\tau, r, \alpha$ etc., of the plectonemic helices are obtained using (19) as shown in section 3 . In the above equations $L_{f}$ is the total length of the DNA in the nearly straight highly 
fluctuating part of the rod while $L_{1}+4 \pi m r \sec \alpha$ is the remaining length where the fluctuations are negligible. From (32) we see that the writhe contribution to the total link comes from both the plectonemes $(2 m \sin \alpha)$ as well as the fluctuations $\left(\frac{M_{3} L_{f} k_{B} T}{4 K_{b} \sqrt{K_{b} F}}\right)$. In fact, when $T=0$ there are no fluctuations so that $L_{f}=0$ and we recover the equations of section 3 . We also note that at $s= \pm \frac{L_{1}}{2}$, which is the junction of the fluctuating part of the rod with the highly curved part, the tangent is continuous ${ }^{2}$ since $Z^{\prime}\left( \pm \frac{L_{1}}{2}\right)=1$. We observe also that $Z^{\prime \prime}\left( \pm \frac{L_{1}}{2}\right)=0$ which together with the enforced continuity of $F, M_{3}$ and $M_{z}$ gaurantees that the force and moment vectors are also continuous at $\pm \frac{L_{1}}{2}$.

Equations (28) to (33) are six equations in the seven unknowns $-s_{1}, z_{1}, z_{3}, M_{3}, m, L_{2}$ and $L_{f}$. We need one more condition to ensure that we have as many equations as there are unknowns. This last equation is obtained from the principle that the free energy of the equilibrium configuration should be a minimum. The calculation of the free energy of a fluctuating rod (with both bending and twisting) under non-linear constraints such as inextensibility and conservation of link (according to the Fuller-White-Calugrenau relation) requires evaluating a difficult functional integral and is a non-trivial exercise. We will not puruse this exercise at present, but instead use the results of Moroz and Nelson (1998) who were able to perform this calculation in the straight pre-buckled state of the rod. Their expressions together with the observation (see Fain, Rudnick and Ostlund, 1997) that the free energy of the plectonemes contains a negligibly small entropic contribution allows us to write the free energy of the post-buckled plectonemic state of the rod in the following way:

$$
G=4 \pi m r \sec \alpha \mathcal{G}_{\text {hel }}+L_{2} \mathcal{G}_{\text {rod }}+L_{f} \mathcal{G}_{f l c}-F\left(Z\left(+\frac{L}{2}\right)-Z\left(-\frac{L}{2}\right)\right),
$$

where the free energy densities $\mathcal{G}_{\text {hel }}, \mathcal{G}_{\text {rod }}$ and $\mathcal{G}_{\text {flc }}$ are given by

$$
\begin{aligned}
\mathcal{G}_{\text {hel }} & =\frac{M_{3}^{2}}{K_{b}}\left(1-Z^{\prime}\left(s_{1}\right)\right)+\frac{M_{3}^{2}}{2 K_{t}}, \\
\mathcal{G}_{\text {rod }} & =\frac{F}{2}\left(1+z_{1}+z_{3}-z_{1} z_{3}\right)+\frac{M_{3}^{2}}{2 K_{t}}+F \frac{Z\left(+\frac{L_{2}}{2}\right)-Z\left(-\frac{L_{2}}{2}\right)}{L_{2}}, \\
\mathcal{G}_{f l c} & =-k_{B} T \frac{P k_{B} T}{K_{b}}\left(1-\frac{1}{4 P}-\frac{1}{64 P^{2}}\right)+\frac{M_{3}^{2}}{2 K_{t}},
\end{aligned}
$$

where $P=\frac{\sqrt{K_{b} F-\frac{M_{3}^{2}}{4}}}{k_{B} T}$. The free energy given by (34) depends on the variables $z_{1}, z_{3}, s_{1}$ etc., for any given values of $\Delta L k, L, T$ and $F$ and material properties $K_{b}$ and $K_{t}$. Our goal is to find the values of $z_{1}, z_{3}, s_{1}, m, M_{3}, L_{2}$ and $L_{f}$ that satisfy equations (28) to (33) and minimise the free energy given by (34). This calculation was performed by first solving equations (28) to (33) for $z_{1}, s_{1}, M_{3}, m, L_{2}$ and $L_{f}$ using Newton's method and assuming $z_{3}$ as a parameter. The free-energy was then determined as a function of $z_{3}$ and the appropriate value of $z_{3}$ chosen according to the principle of minimum free energy. The resulting dependence of the end-to-end distance $Z\left(\frac{L}{2}\right)-Z\left(-\frac{L}{2}\right)$ on the link added at the ends is shown in figure 6 . The plot is similar to figure 5 but there are some important differences. First, the pre-buckling response is no longer a straight line; the effects of thermal fluctuation are clearly apparent in the significant reduction in the end-to-end distance (about $100 \mathrm{~nm}$ for $F=1 \mathrm{pN}$ ). Second, because of thermal

\footnotetext{
${ }^{2}$ Here continuity of a quantity $X(s)$ should be interpreted as equality of the average values $\left\langle X\left(s_{ \pm}\right)\right\rangle$where $\langle\cdot\rangle$ represents an average in a constant temperature ensemble.
} 
softening, buckling is delayed to a larger value of the number of turns added although the critical torque for buckling is $\sqrt{2 K_{b} F}$ in all cases. Third, the slope of the post-buckling (almost) linear response regime is different, again because of thermal softening. The change in extension at buckling is about $200 \mathrm{~nm}$ both in figure 5 and figure 6 . Finally, another experimental observable, the torque $M_{3}$ is plotted in figure 7 . The torque rises linearly with the number of turns before reaching a critical value $\sqrt{2 K_{b} F}$ (at which buckling occurs) and then remains constant at some $M_{3}<\sqrt{2 K_{b} F}$. As a result there is a jump in the torque at buckling. However, the experiments of Deufel et al. (2007) indicate, contrary to our expectation, that the torque plateaus at a constant value after buckling without showing an abrupt jump, at least at $F=10 \mathrm{pN}$. This motivates us to impose $M_{3}=\sqrt{2 K_{b} F}$ as the seventh adhoc ${ }^{3}$ constraint (instead of requiring the free energy to be a minimum) for closing equations (28) to (33) and examine the consequences. The resulting dependence of end-to-end distance on the number of turns applied is depicted in figure 8. We notice that the jump in extension is much smaller with this constraint but the post-buckling response still remains linear albeit with a different slope. Moreover, the curves in figure 8 look qualitatively similar to the experimental data of Strick et al. (1998). This suggests that within the parameters of our model the magnetic tweezer based experiments of Strick et al. $(1996,1998)$ are best simulated by assuming that the post-buckling torque remains constant at $\sqrt{2 K_{b} F}$. However, Strick et al. $(1996,1998)$ and Charvin et al. (2004) do not report the post-buckling torque in the DNA; they only give the extension as a function of the number of turns of the magnet. It is for this reason that we can, at best, only qualitatively compare our results for the extension vs. number of turns with those of Strick et al.

\section{Conclusions}

In this paper we have developed a simple model for the mechanics of plectoneme formation in a fluctuating rod. Our model is inspired by, and shares some common elements with the works of Stump and Fraser (2000) and Fain, Rudnick and Ostlund (1997) but it differs from them in the nature of the predictions it makes about torsional buckling in fluctuating DNA. In particular, our model predicts a sudden change in the end-to-end distance immediately at buckling. The change in end-to-end distance is roughly $200 \mathrm{~nm}$ for a piece of DNA that is $750 \mathrm{~nm}$ long at a temperature of $300 \mathrm{~K}$. This value of $200 \mathrm{~nm}$ for the change in end-to-end distance is nearly constant for forces in the range of $1-3 \mathrm{pN}$. It is, however, dependent on material parameters such as the bending modulus $K_{b}$ and the twisting modulus $K_{t}$ which we have assumed constant at $205 \mathrm{pNnm}^{2}$ and $431 \mathrm{pNnm}^{2}$ respectively throughout this paper since several independent experiments have reported values in this range. The change in the end-to-end distance as well as the post-buckling slope of the response curve depend strongly on the nature of constraints applied to the DNA as well as on the assumed form of the free energy. In this paper we have used an expression for the free energy that has been derived purely by assuming DNA to be an elastic rod while neglecting electrostatic contributions. The latter might become important for plectonemic radii smaller than $3 \mathrm{~nm}$. We have also assumed that the free energy admits an additive decomposition as summarised by (34) which is not strictly true; the alternative is to perform a calculation similar in spirit to that of Moroz and Nelson (1998) and is rather

\footnotetext{
${ }^{3}$ We can either prescribe $\Delta L k$ or $M_{3}$ but not both. Hence we call this an adhoc constraint. However, we also recognise that the constraints in a real experiment will neither correspond to a hard apparatus in which $\Delta L k$ is prescribed (figure 6), nor a soft apparatus in which the torque $M_{3}$ is prescribed (figure 8) - this will depend on the stiffness of the magnetic or optical trap.
} 
cumbersome. Furthermore, we have assumed, for the sake of simplicity, that the helices have an integer number of turns and a constant pitch. Some of these assumptions can be relaxed and we will discuss their consequences in a future article.

\section{Acknowledgements}

We gratefully acknowledge Chris Deufel, Scott Forth and Michelle Wang at Cornell University for sharing their unpublished experimental data with us and for stimulating discussions.

\section{References}

[1] Abramowitz M., and Stegun I. A., 1964. Handbook of Mathematical functions, National Bureau of Standards, Washington D. C.

[2] Charvin G., Allemand J. -F., Strick T. R., Bensimon D., and Croquette V., 2004. Twisting DNA: single molecule studies. Contemporary Physics 45(5), 383-403.

[3] Coleman, B. D. and Swigon, D., 2000. Theory of superocoiled elastic rings with self contact and its application to DNA plasmids. J. Elasticity 60, 173-221.

[4] Coleman, B. D., Swigon, D. and Tobias, I., 2000. Elastic stability of DNA configurations. II. Supercoiled plasmids with self-contact. Phys. Rev. E 61, 759-770.

[5] Deufel, C., Forth, S., Simmons C. R., Dejgosha, S. and Wang, M. D., 2007. Nanofabricated quartz cylinders for angular trapping: DNA superocoiling and torque detection. Nature Methods 4(3), 223-225.

[6] Fain, B., Rudnick, J. and Ostlund, S., 1997. Conformations of linear DNA. Phys. Rev. E 55(6), 7364-7368.

[7] Fuller, F. B., 1971. The writhing number of a space curve. Proc. Natl. Acad. Sci. USA 68, 815-819.

[8] Love, A. E. H., 1944. A Treatise on the Mathematical Theory of Elasticity, Dover, New York.

[9] Maddocks J. H., 1984. Stability of non-linearly elastic rods. Arch. Rat. Mech. Anal. 85, 311-354.

[10] Marko, J. F. and Siggia, E. D., 1995. Stretching DNA. Macromolecules 28, 8759-8770.

[11] Moroz, J. D. and Nelson, P. C., 1997. Torsional directed walks, entropic elasticity and DNA twist stiffness. Proc. Natl. Acad. Sci. USA 94 14418-14422.

[12] Moroz, J. D. and Nelson, P. C., 1998. Entropic elasticity of twist storing polymers. Macromolecules 31, 6333-6347.

[13] Nelson, P. C., 2004. Biological Physics, W. H. Freeman and company, New York.

[14] Neukirch, S., 2004. DNA twist rigidity from experimental supercoiling data. Phys. Rev. Lett. 93(19), 198107. 
[15] Nizette, M. and Goriely, A., 1999. Towards a classification of Euler-Kirchhoff filaments. $J$. Math. Phys. 40(6), 2830-2866.

[16] Odijk, T., 1995. Stiff chains and filaments under tension. Macromolecules 28, 7016-7018.

[17] Purohit, P. K. and Nelson, P. C., 2006. Effect of supercoiling on formation of protein mediated DNA loops. Phys. Rev. E 74, 061907.

[18] Starostin, E. L., 2004. Symmetric equilibria of a thin elastic rod with self-contacts. Phil. Trans. R. Soc. Lond. A 362, 1317-1334.

[19] Strick, T. R., Allemand, J.-F., Bensimon D., Bensimon A. and Croquette V., 1996. The elasticity of a single supercoiled DNA molecule. Science 271, 1835-1837.

[20] Strick, T. R., Allemand, J.-F., Bensimon D., and Croquette V., 1998. Behavior of supercoiled DNA. Biophys. J. 74, 2016-2028.

[21] Stump, D. M. and Fraser, W. B., 2000. Multiple solutions for writhed rods: implications for DNA supercoiling. Proc. R. Soc. Lond. A 456, 455-467.

[22] Stump, D. M., Fraser, W. B. and Gates, K. E., 1998. The writhing of circular cross-section rods: undersea cables to DNA supercoils. Proc. R. Soc. Lond. A 454, 2123-2156.

[23] Thompson, J. M. T., van der Heijden G. H. M. and Neukirch S., 2002. Supercoiling of DNA plasmids: mechanics of the generalized ply. Proc. $R$. Soc. Lond. A 458, 959-985.

[24] van der Heijden, G. H. M., Neukirch, S. and Goss, V. G., 2003. Instability and self-contact phenomena in the writhing of clamped rods. Intl. J. Mech. Sci. 45, 161-196. 


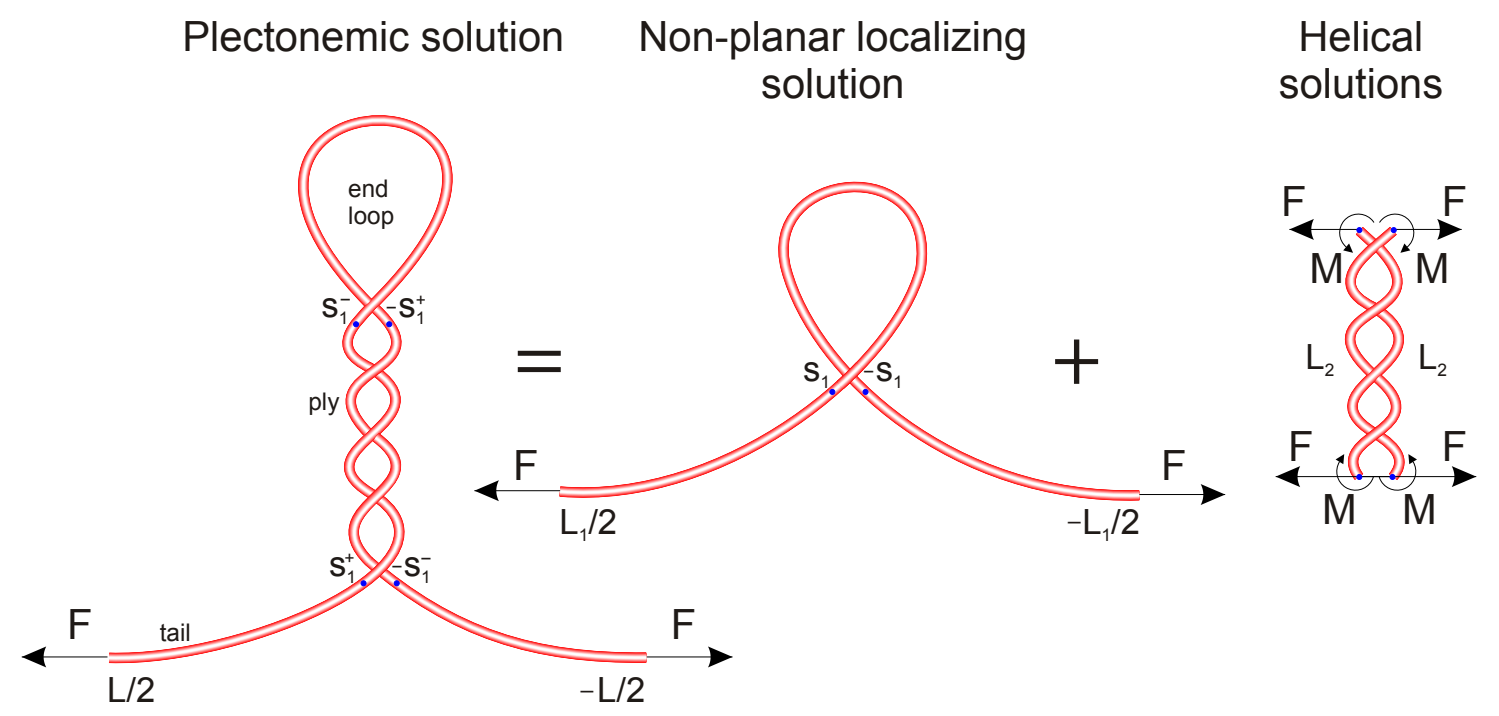

Figure 1: Plectonemic solutions of a twisted rod with no self-contact. The figure illustrates how plectonemic solutions are constructed by combining non-planar localizing solutions (such as the Coyne solution) with the constant curvature helical solutions. The non-planar localizing solution is cut at points $\pm s_{1}$, the end-loop region is separated from the tail region and two helices with an integer number of turns are inserted in such a way that the curvature (or moment), slope and position remain continuous. There is no self-contact anywhere along the plectonemic solution since it is constructed from two other solutions which have no self-contact. Since there is no contact the force remains continuous through out the plectonemic solution. 


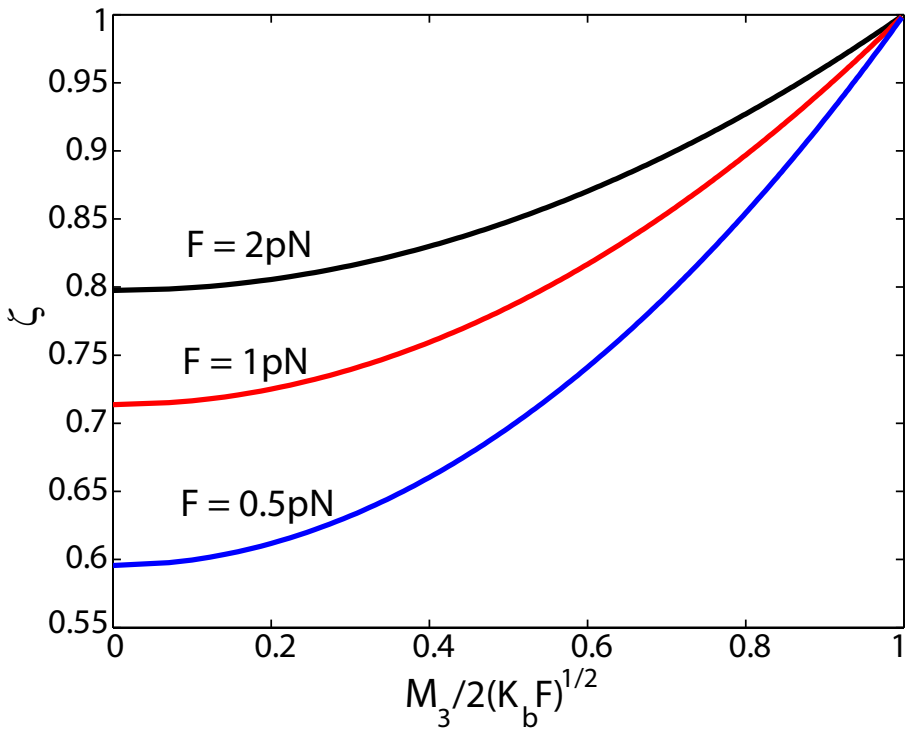

Figure 2: Non-dimensionalized extension $\zeta=\frac{Z(L / 2)-Z(-L / 2)}{L}$ of a buckled rod of length $L=$ $200 \mathrm{~nm}$ plotted as a function of the non-dimensionalized applied torque $\frac{M_{3}}{2 \sqrt{K_{b} F}}$. Three curves for different values of the force $F$ are depicted. The extension at $M_{3}=0$ scales as $F^{-1 / 2}$. As $M_{3} \rightarrow 2 \sqrt{K_{b} F}$ the rod straightens as is evident from the fact that $\zeta \rightarrow 1$ in this limit. The non-planar writhed region (for illustrations of the writhed region in non-planar localizing solutions, see Nizette and Goriely, 1999) becomes smaller and eventually disappears as $M_{3} \rightarrow$ $2 \sqrt{K_{b} F}$. The figure has been constructed assuming mechanical properties $K_{b}=205 \mathrm{pNnm}^{2}$ and $K_{t}=431 \mathrm{pNnm}^{2}$ which are typical of double-stranded DNA. 


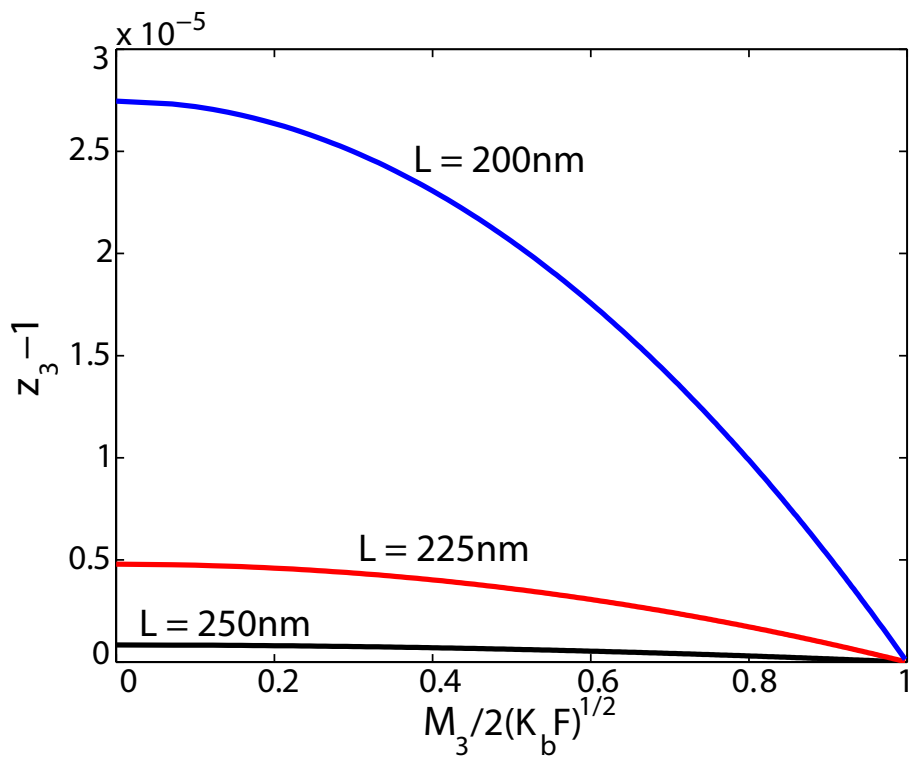

Figure 3: Parameter $z_{3}-1$ plotted as a function of the non-dimensionalized applied torque $\frac{M_{3}}{2 \sqrt{K_{b} F}}$ for three different values of $L$ at $F=1 \mathrm{pN} . z_{3} \approx 1$ for all values of the torque $M_{3}$ and furthermore $z_{3} \rightarrow 1$ as $L$ is increased. Typically, $L=1 \mu \mathrm{m}$ in single molecule experiments on DNA, implying that assuming $z_{3}=1$ would introduce little error in the calculations. The figure has been constructed assuming mechanical properties $K_{b}=205 \mathrm{pNnm}^{2}$ and $K_{t}=431 \mathrm{pNnm}^{2}$ which are typical of double-stranded DNA. 

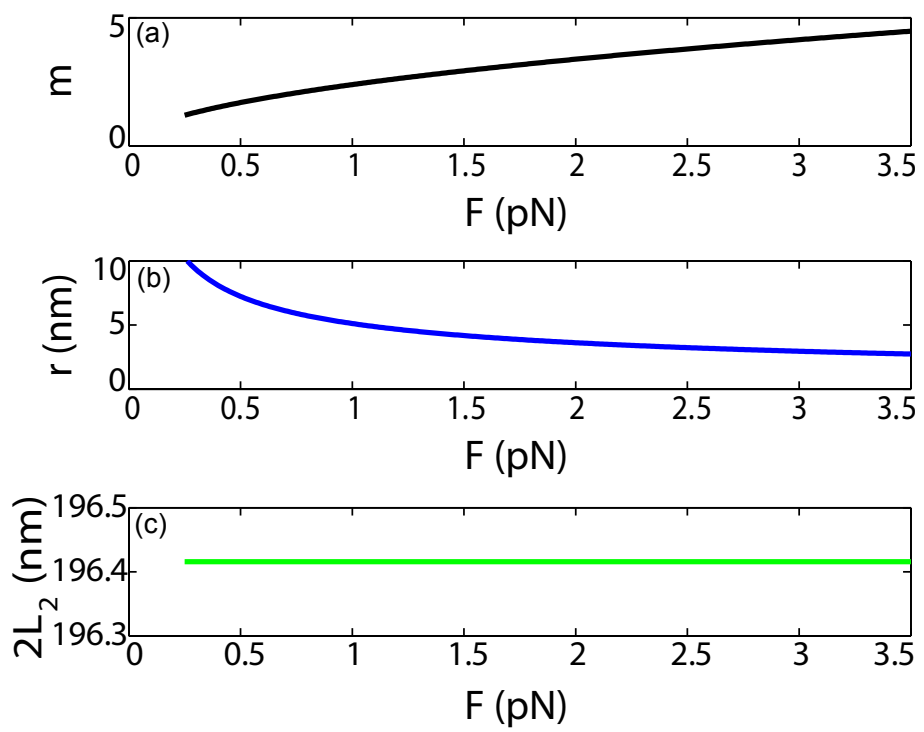

Figure 4: Various parameters of the post-buckling plectoneme plotted as a function of the force $F$ assuming no thermal fluctuations in a DNA fragment of length $L=750 \mathrm{~nm}$. (a) The number of turns $m$ in each of the helices of the plectoneme increases with increasing force. (b) The radius of the helices in the plectoneme decreases with increasing force $\left(r \propto F^{-1 / 2}\right)$. (c) The length $2 L_{2}$ eaten up by the plectoneme remains constant irrespective of the force at $196.4 \mathrm{~nm}$. This also implies that the length $L_{1}=L-2 L_{2} \approx 553 \mathrm{~nm}$, for which the assumption $z_{3}=1$ used to obtain these figures is valid (see figure 3 ). The calculations were performed assuming $K_{b}=205 \mathrm{pNnm}^{2}$ and $K_{t}=431 \mathrm{pNnm}^{2}$ which are typical of double-stranded DNA. 


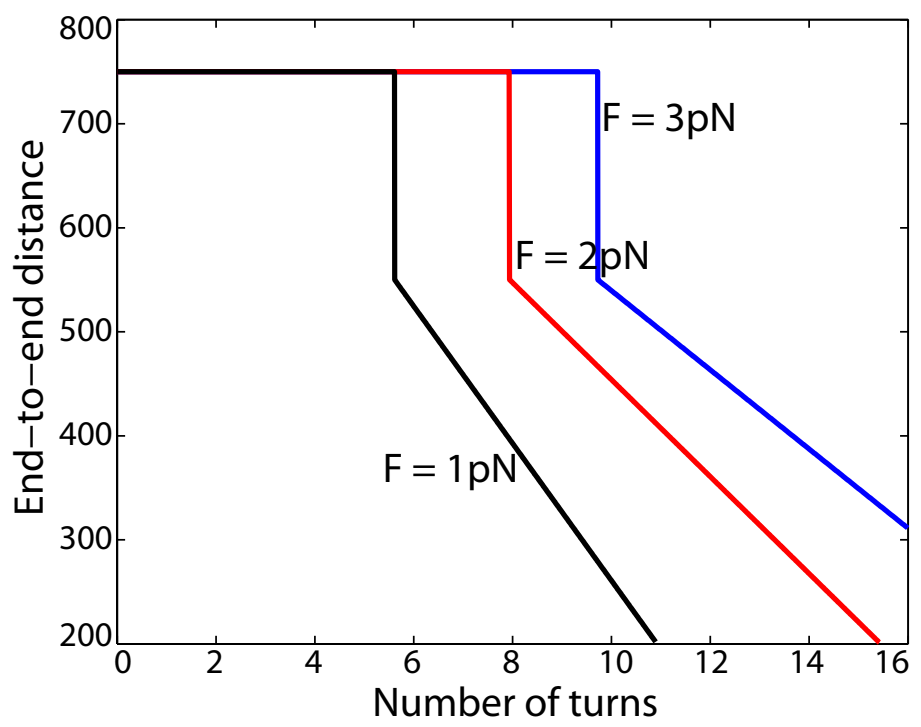

Figure 5: The end-to-end distance of a rod of length $L=750 \mathrm{~nm}$ plotted as a function of the number of turns applied at the end assuming no thermal fluctuations. The rod is assumed to buckle at a critical value of $\Delta L k_{c}=\frac{L \sqrt{2 K_{b} F}}{2 \pi K_{t}}$. If the number of turns applied at the end is less than $\Delta L k_{c}$ the rod remains straight with an end-to-end distance $L$ but after buckling it forms plectonemes which eat up length $2 L_{2}$ hence causing jump in extension at the critical link $\Delta L k_{c}=\frac{L \sqrt{2 K_{b} F}}{2 \pi K_{t}}$. Adding more turns to the rod results in a linear relation of the end-to-end distance vs. number of turns. These plots were generated assuming $K_{b}=205 \mathrm{pNnm}^{2}$ and $K_{t}=431 \mathrm{pNnm}^{2}$ which are typical of double-stranded DNA. 


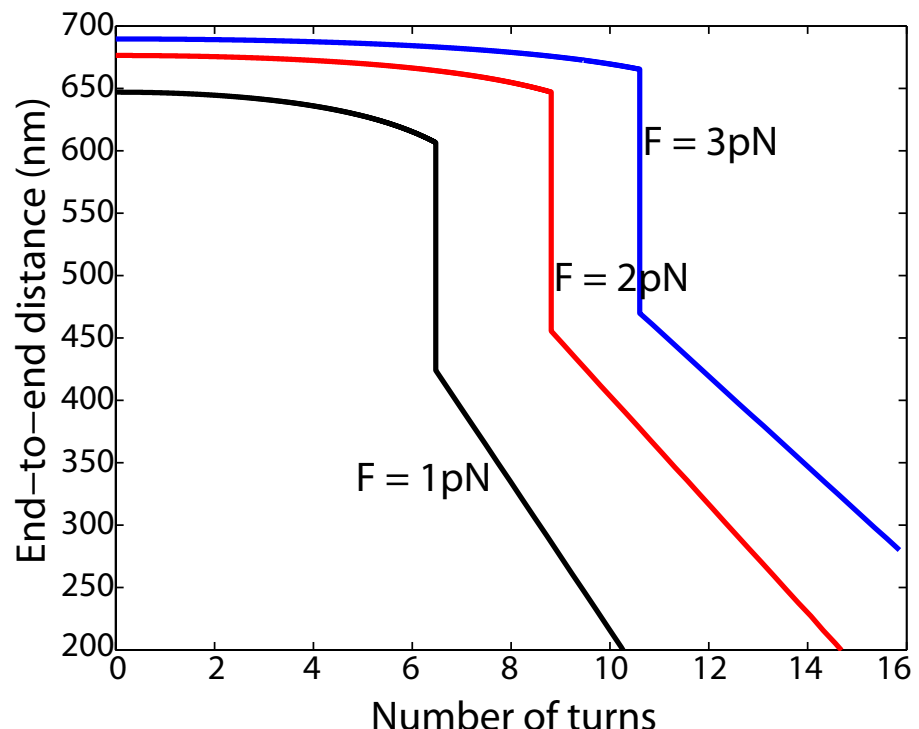

Figure 6: The end-to-end distance of a fluctuating rod of length $L=750 \mathrm{~nm}$ plotted as a function of the number of turns applied at $T=300 \mathrm{~K}$. The rod buckles at a critical value of the torque $M_{c}=\sqrt{2 K_{b} F}$. If the twisting moment applied to the rod is less than this critical value it fluctuates about a straight configuration and the end-to-end distance is determined from the theory of Moroz and Nelson (1997). The end-to-end distance is smaller than $L$ because thermal fluctuations 'eat up' some length. The eaten-up length is lesser if the tension $F$ is larger. After buckling the rod forms plectonemes which eat up some more length $2 L_{2}$ hence causing a jump in extension at the critical torque $\sqrt{2 K_{b} F}$. Adding more turns to the rod results in an almost linear relation of the end-to-end distance vs. number of turns. These plots were generated assuming $K_{b}=205 \mathrm{pNnm}^{2}$ and $K_{t}=431 \mathrm{pNnm}^{2}$ which are typical of double-stranded DNA. 


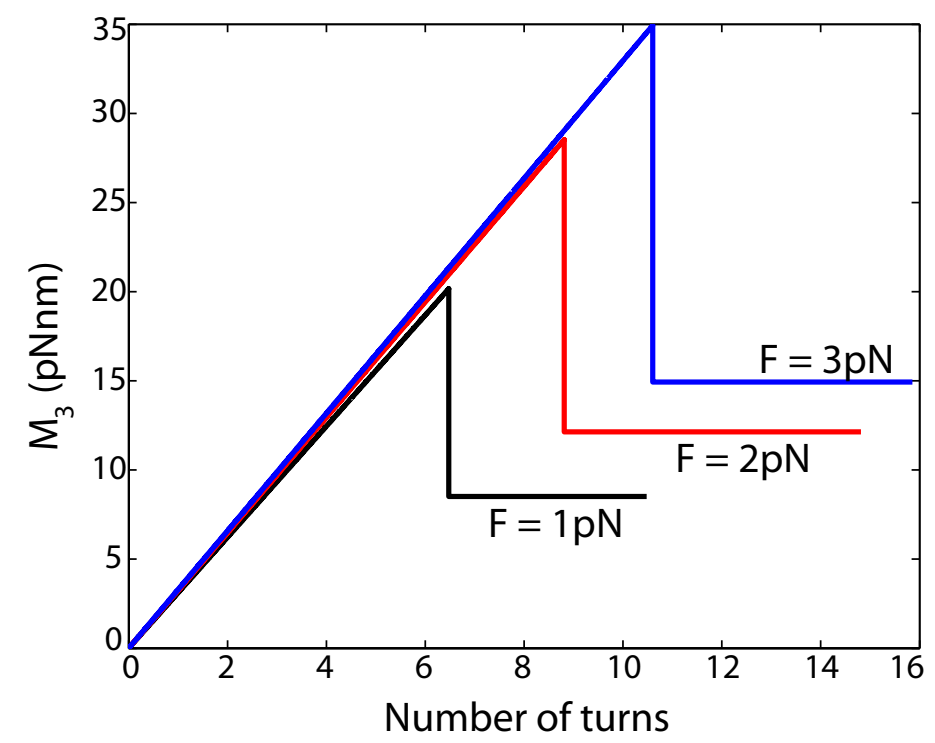

Figure 7: The twisting moment $M_{3}$ for a fluctuating rod of length $750 \mathrm{~nm}$ plotted as a function of the number of turns. The pre-buckling torque is linear in the number of turns while the post-buckling torque is constant at some value $M_{3}<\sqrt{2 K_{b} F}$. This, however, is not observed in the experiments of Deufel et al. (2007); the post-buckling torque is constant at $\sqrt{2 K_{b} F}$ and does not show an abrupt jump. We impose this as a constraint and plot the end-to-end distance vs. number of turns in figure 8 .

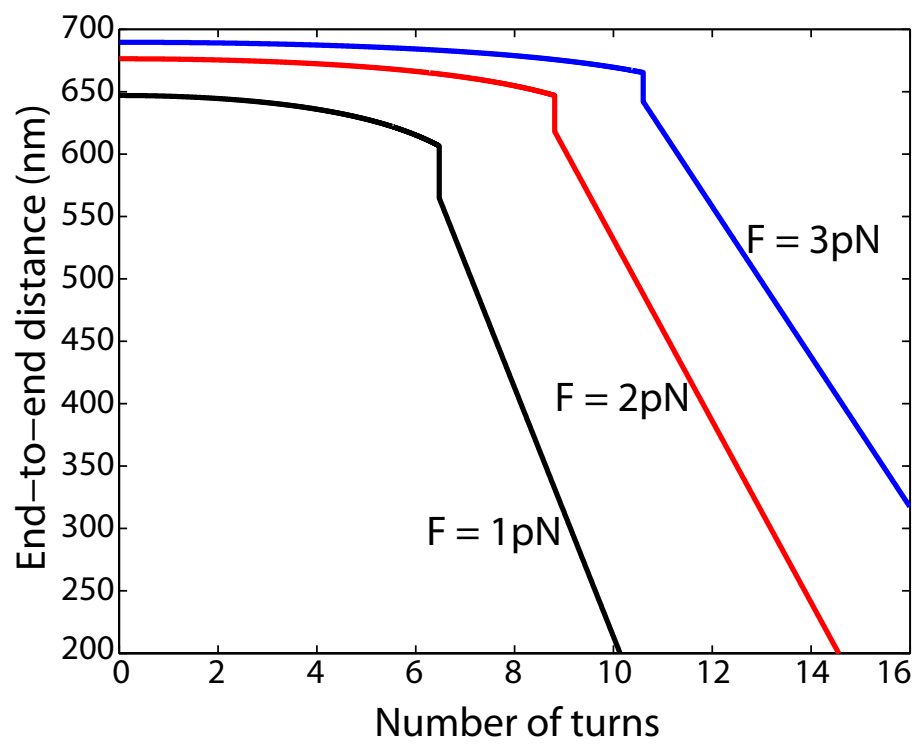

Figure 8: The end-to-end distance of a fluctuating rod of length $L=750 \mathrm{~nm}$ plotted as a function of the number of turns applied at $T=300 \mathrm{~K}$ under the constraint that the post-buckling torque is $\sqrt{2 K_{b} F}$. This figure is to be contrasted with figure 6 which is based on the minimisation of free energy. The change in extension at buckling is significantly lesser in the present case. As a result these curves look qualitatively similar to the experimental data of Strick et al. $(1996,1998)$. 